\title{
Axillary Keloid Formation after Osmidrosis Surgery
}

\author{
So-Min Hwang, Sang-Hwan Lee, \\ Hyung-Do Kim, Yong-Hui Jung, \\ Hong-il Kim
}

Aesthetic, Plastic \& Reconstructive Surgery Center, Good Moonhwa Hospital, Busan, Korea

This study was presented at the 3rd Research \& Reconstructive Forum on May 9-10, 2013, in Daegu, Korea.

No potential conflict of interest relevant to this article was reported.
Keloid is a scar tissue that undergoes recovery and excessive growth over the original wounds when the collagen is excessively accumulated in the dermis during the wound healing process. The common sites of keloid occurrence include the anterior thorax, shoulder, upper extremities and ear. To our knowledge, however, there are few cases of keloid that occurs in the axilla. In addition, there are fewer cases of keloid that bilaterally occurs at the operated sites postoperatively in individuals with no past or family history. 21-year-old male had undergone subdermal shaving for the management of osmidrosis but had not received appropriate scar management during the military service. He visited us with a keloid-like scar formed in the bilateral axillae. We excised the scar tissue and treated it with local steroid injection and postoperative axilla compression. In histopathological report, no flattening of the overlying epidermis, and presence of keloid collagen which confirms diagnosis of keloid. We report our clinical experience with a rare case of keloid occurring in the axilla and treatment process.

Keywords Apocrine glands, Keloid, Scar

\section{INTRODUCTION}

Keloid is a scar tissue that undergoes recovery and excessive growth over the original wounds when the collagen is excessively accumulated in the dermis during the wound healing process. Unlike hypertrophic scar that is limited to the sites of injury and achieves a recovery over time, keloid is characterized by no changes in the size over time [1]. It may occur with no respect to the age and shows a predilection in individuals aged between 10 and 30 years. The causative factors include skin injuries due to surgery, laceration, tattooing, injections and prophylactic vaccination, excessive skin tension and hormonal imbalance [2]. It commonly affects individuals with genetic predisposition. The common sites of occurrence include the anterior thorax, shoulder, upper extremities and

Received: Sep 1, 2013 Revised: Oct 12, 2013 Accepted: Oct 18, 2013

Correspondence: So-Min Hwang Aesthetic, Plastic \& Reconstructive Surgery Center, Good Moonhwa Hospital, 119 Beomil-ro, Dong-gu, Busan 601-803, Korea. E-mail:Sangwind@hanmail.net

http://submit.e-aaps.org/

Copyright (๑) 2013 The Korean Society for Aesthetic Plastic Surgery.

This is an Open Access article distributed under the terms of the Creative Commons Attribution Non-Commercial License (http://creativecommons.org/licenses/by-nc/3.0/) which permits unrestricted non-commercial use, distribution, and reproduction in any medium, provided the original work is properly cited ear [3] To our knowledge, however, there are few cases of keloid that occurs in the axilla. In addition, there are fewer cases of keloid that bilaterally occurs at the operated sites postoperatively in individuals with no past or family history. But, we experience with a rare case of keloid occurring in the axilla after osmidrosis surgery and then we report our case with a review of literatures.

\section{CASE}

A 21-year-old man visited us with a chief complaint of a keloidlike scar formed in the bilateral axillae. On history taking, the patient underwent subdermal shaving two years ago for the management of osmidrosis but had not received appropriate scar management during the military service. The mass had occurred six months before outpatient visit and then it had slowly grown, for which the patient had not received any appropriate treatments. At the time of outpatient visit, the patient complained of pruritis at the sites of scar formation. But the patient presented with no other specific symptoms. On family or past history, the patient had no notable findings associated with keloid. Moreover, the patient also had no notable findings at a preoperative work-up. On physical examination, the patient had a tenderness and he also had a glossy, pinkcolored, firm, oval-shaped, well-defined mass of $5.0 \times 1.5 \times 0.5 \mathrm{~cm}$ in size in the right axilla and $5.0 \times 2.0 \times 1.0 \mathrm{~cm}$ in size in the left 

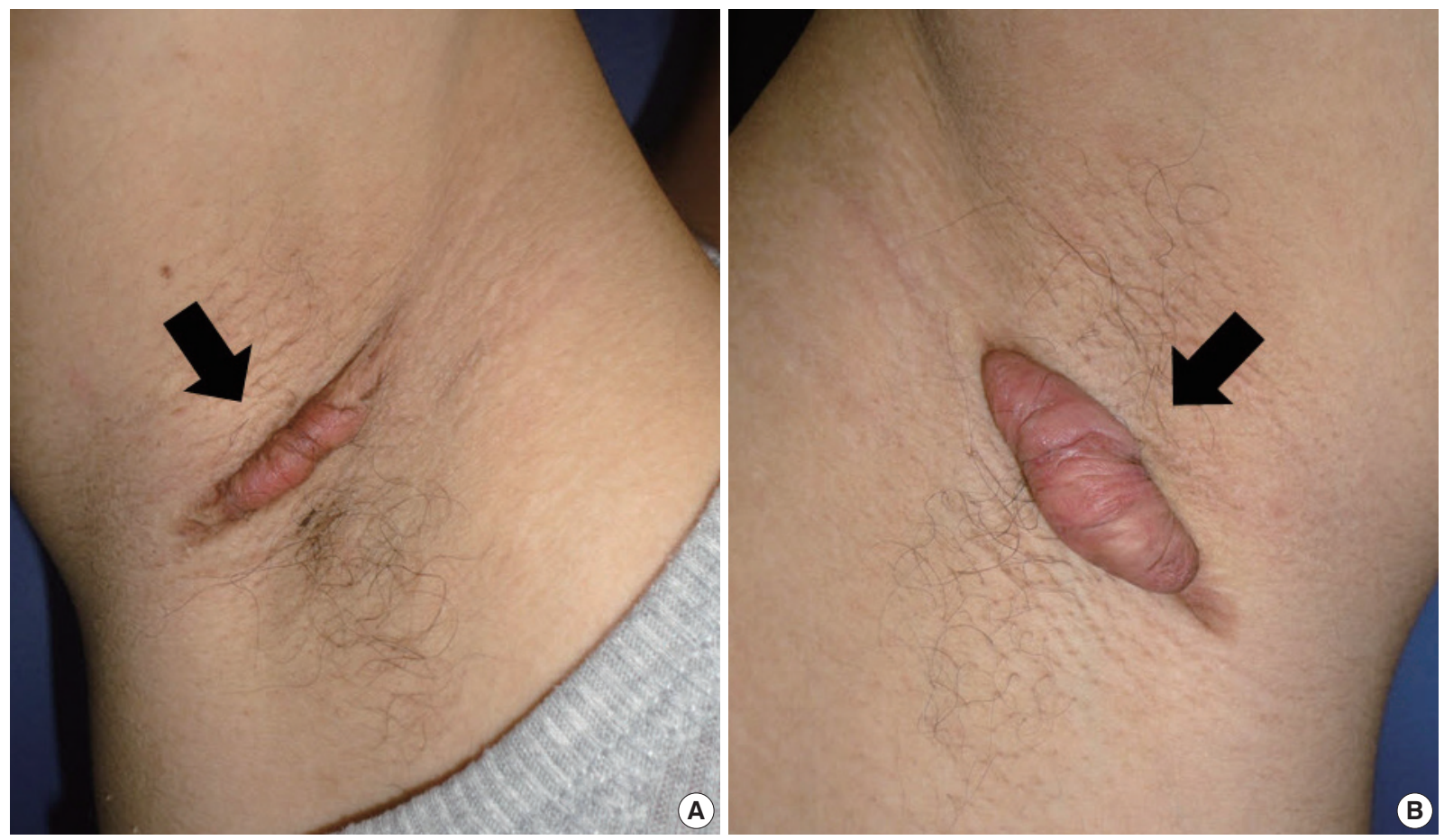

Fig. 1. Preoperative clinical photographs show a firm and oval-shaped mass (black arrows). (A) The mass of $5.0 \times 1.5 \times 0.5 \mathrm{~cm}$ in size in the right axilla. (B) The mass of $5.0 \times 2.0 \times 1.0 \mathrm{~cm}$ in size in the left axilla.

axilla (Fig. 1). Based on the physical examination and the morphology of the mass, we assumed that it would be highly probable that the mass might be a keloid-like scar that occurred postoperatively. We therefore planned combination treatments of surgery with steroid injections. Under intravenous anesthesia, we performed intralesional excision. This procedure leaves the outer segment of the keloid, cutting out the central portion of the scar. At the time of surgical excision, the wound bed and margins in the bilateral regions, were injected with a small amount of intradermal triamcinolone acetonide $40 \mathrm{mg} / \mathrm{mL}$ diluted to $10 \mathrm{mg} / \mathrm{mL}$. To compress the operated sites, the gauze was applied in a fluffy ball shape on both sides of the axilla and followed by dressing using an elastic bandage. On gross finding, cross sections of the mass contain homogeneous fibrous materials. In histopathological report, no flattening of the overlying epidermis, and presence of keloid collagen which confirms diagnosis of keloid (Fig. 2). During the regular follow-up period, the patient was given steroid injections twice more at a 3-week interval. Three years postoperatively, the patient currently presented with no recurrence or other notable episodes (Fig. 3).

\section{DISCUSSION}

Keloid was first described in the days of the ancient Egyptian empire. In addition, it was first coined, by Alibert in 1806, to describe its overgrowth into the adjacent normal tissue (from the Greek

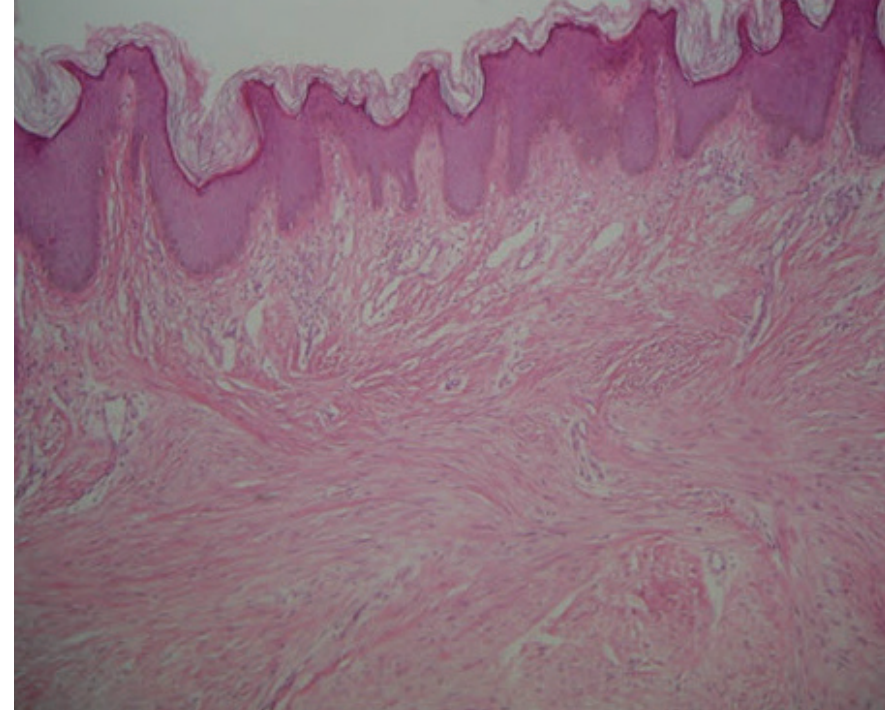

Fig. 2. Histopathologic finding. An irregular, thick, glassy and dense collagen bundles observed $(H \& E, \times 40)$.

chele, meaning "crab's claw") [4]. Currently, it is referred to as fibrous hyperplasia after the onset of skin trauma due to various etiologic factors. The causative factors include skin injuries due to surgery, laceration, tattooing, injections and prophylactic vaccination, excessive skin tension and hormonal imbalance. Still, however, little is known about the exact mechanisms by which the keloid is formed [2]. It has been hypothesized that it occurs as a re- 

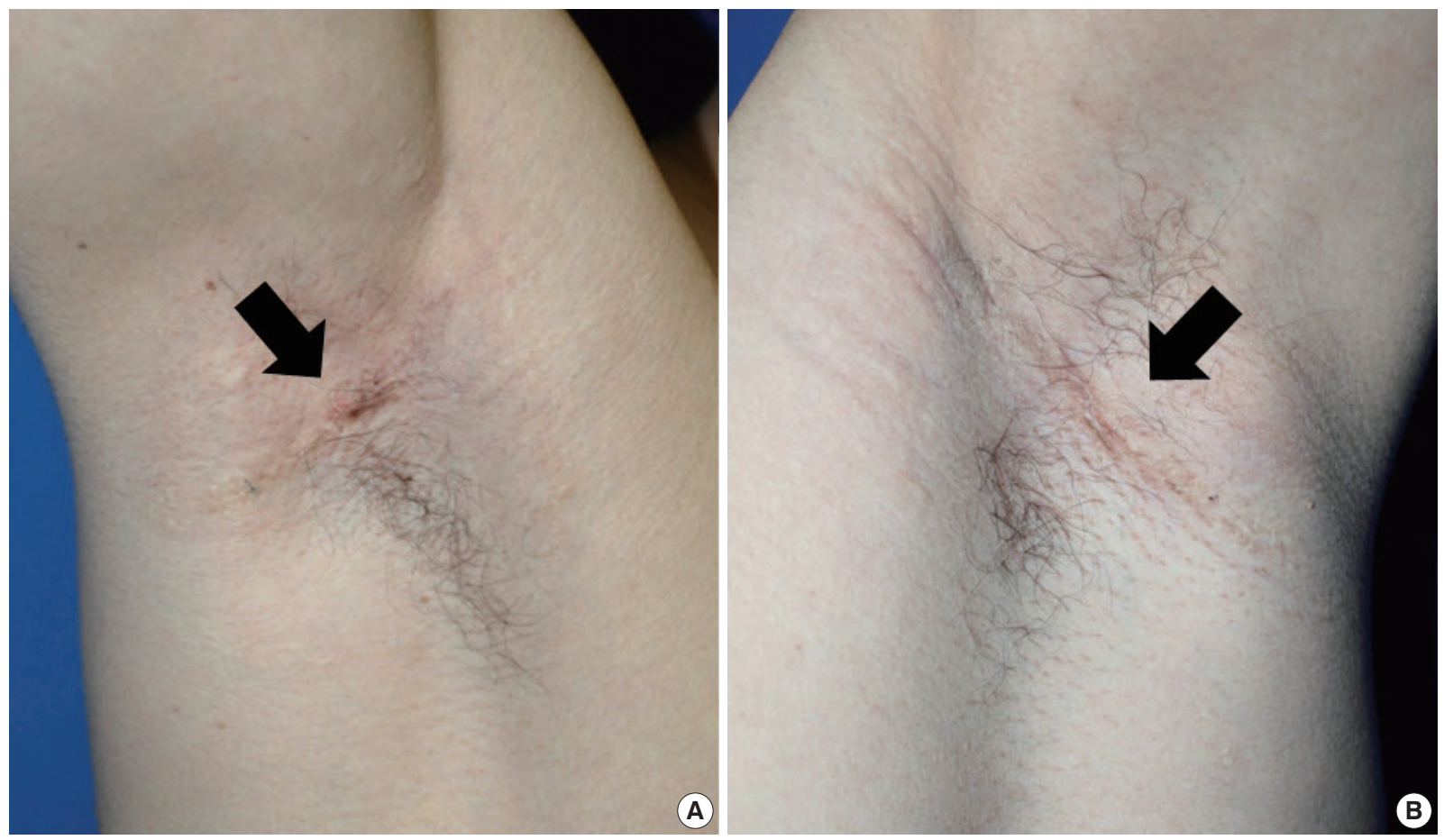

Fig. 3. Postoperative clinical photographs. Clinical photographs at postoperative 3 years after keloidectomy and triamcinolone injection showing no evidence of recurrence in the right (A) and left (B) axilla (arrows).

sult of the derangement in the growth factor control, abnormal collagen synthesis, abnormal immune response and the abnormal functions of the sebaceous gland.

Once developed, keloid shows a high rate of recurrence and causes many cosmetic problems because no complete cure can be achieved. It is therefore important to make a differential diagnosis between keloid and hypertrophic scar. Keloid can be differentially diagnosed from hypertrophic scar based on the clinical and histopathologic characteristics. Hypertrophic scar is characterized by its location limited to the initial wounds and occurs within the first four weeks after the onset of the injury. But keloid shows excessive growth over the initial wounds and is formed after four weeks from the onset of the injury. Moreover, hypertrophic scar is decreased in size within one year. But keloid is not decreased in size for more than one year [1]. Histopathologic examinations show that both keloid and hypertrophic scar are characterized by the increased fibrous bundles. However, keloid is characterized no flattening of the overlying epidermis, no scarring of the papillary dermis, keloid-like collagen fibers, fibrous bands like fascia and the irregular arrangement of fibrous bundle. In the current case, the mass had the histopathologic findings that are suggestive of keloid; these include abundant collagen fibers and the irregular arrangement of fibrous bundle. But it occurred in the axilla rather than the anterior chest, shoulder, upper extremities and ear, all of which are considered the common sites of occurrence of keloid. To our knowledge, our case is rare in that it occurred bilateral ax- illae to the operated sites despite a lack of both a past and a family history. Then, we assume that the causes of keloid as shown below:

1) The patient could not receive the treatments for wounds after undergoing surgery for osmidrosis during the military service. It is therefore highly probable that the inflammations might have occurred at the operated sites.

2) The patient did not receive the appropriate treatments for the inflammatory responses. But we experience limitations in identifying the causes of keloid in the current case.

As the treatment modalities for keloid, surgery has been used the most commonly in patients with keloid. It has been reported, however, that surgery alone causes a higher rate of recurrence of $45-100 \%$ [5]. Therefore, local steroid injections are frequently used as adjuvant therapy to surgery for the purposes of inhibiting the expression of collagen genes in patients with keloid [6]. Chowdri et al. reported that the recurrence rate was $8.1 \%$ during a followup period of 30 months following a concomitant use of surgery with local steroid injections, thus advocating the efficacy of the combination of the two methods [7]. It can therefore be stated that the combination of surgical excision and local steroid injections is currently the most popular modality. Although the keloid was effectively removed, it is characterized by long-term recurrence. Therefore, the follow-up period would be a key factor. It has been reported that 5.5-12.9 months elapsed on average until the recurrence following the treatment [7]. At our institution, there were no notable episodes such as tissue atrophy, telangiectasia for- 
mation, hypopigmentation, and recurrence during a 3-year follow-up period.

As shown in our case, there is a possibility that keloid might occur in the axilla. Therefore, keloid formation after axillary operation deserves special attention. In the current case, keloid was formed after approximately one year and six months postoperatively. It can therefore be inferred that both short-term treatments of the operated sites and a long-term regular follow-up would be essential. In addition, although unclear, insufficient or inappropriate postoperative management would lead to the formation of keloid scar. Then, postoperative care also deserves special attention.

We experienced a rare case of keloid occurring in the axilla and then successfully treated it with intralesional excision and local steroid injections. Here, we report our case with a review of literatures.

\section{REFERENCES}

1. Murray JC. Scars and keloids. Dermatol Clin 1993;11:697-708.

2. Al-Attar A, Mess S, Thomassen JM, et al. Keloid Pathogenesis and treatment. Plast Reconstr Surg 2006;117:286.

3. De Felice B, Wilson RR, Nacca M, et al. Molecular characterization and expression of p63 isoforms in human keloids. Mol Genet Genomics 2004;272:28-34.

4. Alibert JLM. Note sur la keloide. J Univ Sci Med 1816;2:207-16.

5. Mustoe TA, Cooter RD, Gold MH, et al. International clinical recommendations on scar management. Plast Reconstr Surg 2002;110:560-71.

6. Griffith BH, Monroe CW, Mckinney P. A follow-up study on the treatment of keloids with triamcinolone acetonide. Plast Reconstr Surg 1970;46:145-50.

7. Chowdri NA, Masarat M, Mattoo A, et al. Keloids and hypertrophic scars: Results with intraoperative and serial postoperative corticosteroid injection therapy. Aust N Z J Surg 1999;69: 655-9. 